\title{
Octonion Electrodynamics in Isotropic and Chiral medium
}

\author{
B. C. Chanyal ${ }^{(1)}$, P. S. Bisht ${ }^{(1)}$, Tianjin $\mathrm{Li}^{(2)}$ and O. P. S. Negi ${ }^{(1)}$ \\ (1) Department of Physics, \\ Kumaun University, \\ S. S. J. Campus, \\ Almora-263601 (Uttarakhand) \\ India
}

(2) Institute of Theoretical Physics, Chinese Academy of Sciences, P. O. Box 2735, Beijing 100080, P. R. China

\author{
Email - bcchanyal@gmail.com \\ ps_bisht 123@rediffmail.com \\ tli@itc.ac.cn \\ ops_negi@yahoo.co.in
}

\begin{abstract}
Starting with the Dirac-Maxwell's equations in presence of electric and magnetic sources in an isotropic medium of dyons, we have derived the generalized octonion Maxwell's equations in isotropic medium. And the octonion formulation of generalized electromagnetic fields in chiral medium has also been developed in compect, simple and consistent manner.
\end{abstract}

Key Words: Octonions, dyons, isotropic and chiral medium, GDM equations etc.

\section{Introduction}

There has been a revival in the formulation of natural laws so that there exists [1] four division algebras consisting the algebra of real numbers, complex numbers, quaternions and octonions. Octonion analysis has been widely discussed by Baez [2]. It has also played an important role in the context of various physical problems of higher dimensional supersymmetry, supergravity and super strings etc. Few interest in the subject of monopoles and dyons was enhanced by the work of t' Hooft [3] and Polyakov [4] and its extension by Julia and Zee [5]. keeping in view the potential importance of monopole and the results of Witten [6] that monopoles are necessarily dyon, Bisht et. al. [7, 8] constructed a self-consistent co-variant theory of generalized electromagnetic fields associated with dyons each carrying the generalized charge as complex quantity with its real and imaginary part as electric and magnetic constituents. Kravchenko and co-authers [9, 10] discussed the Maxwell's equations in homogeneous media and accordingly developed [11] the quaternionic reformulation of the time-dependent Maxwell's equations along with the classical solution of a moving source i.e. electron. Kravchenko 
et al have also demonstrated [12] the electromagnetic fields in chiral media and their quaternionic form in a simple and consistent manner. Recently, Bisht et. al. have developed the generalized Dirac Maxwell's equations in homogeneous (isotropic) medium [13] while their quaternionic forms in a unique and consistent way are also developed [14]. They have already obtained the solution of time independent generalized Dirac Maxwell's (GDM) equations in presence of electric and magnetic sources in chiral media and inhomogeneous media [14][15]. The quaternionic reformulation of generalized electromagnetic fields of dyons in chiral and inhomogeneous media has also been developed and the corresponding quaternionic equations are derived in compect, simple and consistent manner [15]. In this paper we have derived the various quantum equations of generalized electromagnetic fields of dyons (particles carrying simultaneously electric and magnetic charge) in isotropic medium in consistent and manifest co-variant ways. It has been shown that the present theory of dyons remains invariant under the duality transformations in isotropic homogeneous and chiral medium. The octonions analysis of time dependent Maxwell's equations in presence of electric and magnetic charges are obtained in unique, simple and consistent manner.

\section{Generalized Electrodynamic of Dyons in Isotropic Medium}

In order to write the various quantum equations of dyons in isotropic medium, we start with the definition of homogeneous (isotropic) medium [10-13] in the generalized electromagnetic fields as,

$$
\begin{array}{ll}
\vec{D}=\epsilon \overrightarrow{\mathcal{E}} & \left(\epsilon=\epsilon_{0} \epsilon_{r}\right), \\
\overrightarrow{\mathcal{B}}=\mu \vec{H} & \left(\mu=\mu_{0} \mu_{r}\right)
\end{array}
$$

where $\vec{D}$ and $\overrightarrow{\mathcal{B}}$ are respectively the electric and magnetic induction vectors while $\overrightarrow{\mathcal{E}}$ and $\vec{H}$ are generalized electromagnetic fields. Here $\epsilon_{0}$ is the free space permittivity, $\mu_{0}$ is the permeability of free space, $\epsilon_{r}$ and $\mu_{r}$ are defined respectively as relative permittivity and permeability associated with electric and magnetic fields. Applying the above relations in Dirac [16] Maxwell's field equations and establish the dual invariance between electric and magnetic in isotropic medium as [15],

$$
\begin{aligned}
\vec{\nabla} \cdot \overrightarrow{\mathcal{E}} & =\frac{\rho}{\epsilon} \\
\vec{\nabla} \cdot \overrightarrow{\mathcal{B}} & =\mu \varrho ; \\
\vec{\nabla} \times \overrightarrow{\mathcal{E}} & =-\frac{\partial \overrightarrow{\mathcal{B}}}{\partial t}-\frac{\vec{k}}{\epsilon} \\
\vec{\nabla} \times \overrightarrow{\mathcal{B}} & =\frac{1}{v^{2}} \frac{\partial \overrightarrow{\mathcal{E}}}{\partial t}+\mu \vec{j}
\end{aligned}
$$

where $\rho$ and $\varrho$ are respectively the electric and magnetic charge densities while $\vec{j}$ and $\vec{k}$ are the corresponding current densities. The electric and magnetic fields of dyons are expressed in following differential form in isotropic medium in term of two four - potentials [15] i.e. 


$$
\begin{aligned}
\overrightarrow{\mathcal{E}} & =-\frac{\partial \vec{A}}{\partial t}-\vec{\nabla} \phi-\vec{\nabla} \times \vec{B} ; \\
\overrightarrow{\mathcal{B}} & =-\frac{1}{v^{2}} \frac{\partial \vec{B}}{\partial t}-\vec{\nabla} \varphi+\vec{\nabla} \times \vec{A}
\end{aligned}
$$

where $\left\{A^{\mu}\right\}=\{\phi, v \vec{A}\}$ and $\left\{B^{\mu}\right\}=\{v \varphi, \vec{B}\}$ are the two four - potentials associated with electric and magnetic charges. Defining the complex vector field $\vec{\psi}$ as

$$
\vec{\psi}=\overrightarrow{\mathcal{E}}-i v \overrightarrow{\mathcal{B}}
$$

using the equations $(3)$ and $(4)$, we get the following relations between generalized field $\vec{\psi}$ and the components of four potential as

$$
\vec{\psi}=-\frac{\partial \vec{V}}{\partial t}-\vec{\nabla} \Phi-i v(\vec{\nabla} \times \vec{V})
$$

where $\left\{V_{\mu}\right\}$ is the generalized four - potential of dyons in isotropic medium and is defined as

$$
V_{\mu}=\{\Phi, \vec{V}\}
$$

where

$$
\Phi=\phi-i v \varphi
$$

and

$$
\vec{V}=\vec{A}-\frac{i}{v} \vec{B}
$$

Maxwell's field equations 22 may then be written in term of generalized field $\vec{\psi}$ as

$$
\begin{aligned}
\vec{\nabla} \cdot \vec{\psi} & =\frac{\rho}{\epsilon} \\
\vec{\nabla} \times \vec{\psi} & =-i v\left(\mu \vec{J}+\frac{1}{v^{2}} \frac{\partial \vec{\psi}}{\partial t}\right)
\end{aligned}
$$

where $\boldsymbol{\rho}$ and $\vec{J}$ are the generalized charge and current source densities of dyons in isotropic medium, given as 


$$
\begin{aligned}
\boldsymbol{\rho} & =\left(\rho-i \frac{\varrho}{v}\right) \\
\vec{J} & =(\vec{j}-i v \vec{k}) .
\end{aligned}
$$

Here we introduce a new parameter $\vec{S}$ (called field current) in the following form in term of source densities i. e.

$$
\vec{S}=\square \vec{\psi}=-\mu \frac{\partial \vec{J}}{\partial t}-\frac{1}{\epsilon} \vec{\nabla} \boldsymbol{\rho}-i v \mu(\vec{\nabla} \times \vec{J})
$$

where $\square$ is the D' Alembertian operator. In term of complex potential the Generalized Dirac - Maxwell's (GDM) equations of dyons in isotropic medium are written in the following form

$$
\begin{aligned}
& \square \Phi=v \mu \boldsymbol{\rho} ; \\
& \square \vec{V}=\mu \vec{J} .
\end{aligned}
$$

\section{Generalized Octonionic Maxwell's Equations in Isotropic Medium}

An octonion $\mathcal{O}$ is expressed as [17] a set of eight real numbers

$$
\begin{aligned}
\mathcal{O} & =\mathcal{O}_{0} e_{0}+\mathcal{O}_{1} e_{1}+\mathcal{O}_{2} e_{2}+\mathcal{O}_{3} e_{3}+\mathcal{O}_{4} e_{4}+\mathcal{O}_{5} e_{5}+\mathcal{O}_{6} e_{6}+\mathcal{O}_{7} e_{7} \\
& =\mathcal{O}_{0} e_{0}+\sum_{A=1}^{7} \mathcal{O}_{A} e_{A} . \quad(A=1,2, \ldots ., 7)
\end{aligned}
$$

where $e_{A}(A=1,2, . ., 7)$ are imaginary octonion units and $e_{0}$ is the multiplicative unit element.

The octet $\left(e_{0}, e_{1}, e_{2}, e_{3}, e_{4}, e_{5}, e_{6}, e_{7}\right)$ are known as the octonion basis and its elements satisfy the following multiplication rules

$$
e_{0}=1, \quad e_{0} e_{A}=e_{A} e_{0}=e_{A}, \quad e_{A} e_{B}=-\delta_{A B} e_{0}+f_{A B C} e_{C} . \quad(A, B, C=1,2, \ldots \ldots .7)
$$

The structure constants $f_{A B C}$ are completely antisymmetric and take the value 1 i. e. $f_{A B C}=+1=(123),(471),(257)$, (165), (624), (543), (736).

In order to write the quantum equations of dyons in isotropic media in terms of compact notations of octonions, let us start with the four dimensional representation of differential operator [17, 18] expressed in terms of octonion units as 


$$
\bullet=e_{1} \frac{\partial}{\partial x}+e_{2} \frac{\partial}{\partial y}+e_{3} \frac{\partial}{\partial z}-\frac{i}{v} e_{7} \frac{\partial}{\partial t}
$$

where the other components like $\partial_{0}, \partial_{4}, \partial_{5}, \partial_{6}$ are vanishing. The octonion conjugate differential operator in isotropic medium is defined as

$$
\bar{\nabla}=-e_{1} \frac{\partial}{\partial x}-e_{2} \frac{\partial}{\partial y}-e_{3} \frac{\partial}{\partial z}+\frac{i}{v} e_{7} \frac{\partial}{\partial t}
$$

from equations $(19)$ and 20 we get

$$
\begin{aligned}
\bullet \bar{\ominus} & =\frac{\partial^{2}}{\partial x^{2}}+\frac{\partial^{2}}{\partial y^{2}}+\frac{\partial^{2}}{\partial z^{2}}-\frac{1}{v^{2}} \frac{\partial^{2}}{\partial t^{2}} \\
& =\nabla^{2}-\frac{1}{v^{2}} \frac{\partial^{2}}{\partial t^{2}}=\square=\square \square .
\end{aligned}
$$

In terms of the components of electric $\left\{A_{\mu}\right\}$ and magnetic $\left\{B_{\mu}\right\}$ four - potentials of dyons (particles carrying simultaneously the electric and magnetic charges), the octonionic potential is expressed in the isotropic medium as [17, 18],

$$
\begin{aligned}
\mathbb{V} & =e_{1}\left(A_{x}+i e_{7} \frac{B_{x}}{v}\right)+e_{2}\left(A_{y}+i e_{7} \frac{B_{y}}{v}\right)+e_{3}\left(A_{z}+i e_{7} \frac{B_{z}}{v}\right)+\left(\varphi+i e_{7} \frac{\phi}{v}\right) \\
& =e_{1} \mathrm{~V}_{\mathrm{x}}+e_{2} \mathrm{~V}_{\mathrm{y}}+e_{3} \mathrm{~V}_{\mathrm{z}}+i e_{7} \Phi .
\end{aligned}
$$

where $\left(\Phi, \mathrm{V}_{\mathrm{x}}, \mathrm{V}_{\mathrm{y}}, \mathrm{V}_{\mathrm{z}}\right)=(\Phi, \overrightarrow{\mathrm{V}})=\left\{\mathrm{V}_{\mu}\right\}$ are described as the components of generalized four - potential $\left\{\mathrm{V}_{\mu}\right\}$ associated with dyons in presence of generalized charge $(q=e+i v g)$ (where $e$ and $g$ are respectively known as electric and magnetic charges) of dyons. Now operating $\bar{\nabla}$ to octonion potential $\mathbb{V}$, we get

$$
\begin{aligned}
\varpi \mathbb{V} & =e_{1}\left(-\frac{\partial \varphi}{\partial x}+\frac{\partial A_{z}}{\partial y}-\frac{\partial A_{y}}{\partial z}-\frac{1}{v^{2}} \frac{\partial B_{x}}{\partial t}\right)+e_{2}\left(-\frac{\partial \varphi}{\partial y}+\frac{\partial A_{x}}{\partial z}-\frac{\partial A_{z}}{\partial z x}-\frac{1}{v^{2}} \frac{\partial B_{y}}{\partial t}\right) \\
& +e_{3}\left(-\frac{\partial \varphi}{\partial z}+\frac{\partial A_{y}}{\partial x}-\frac{\partial A_{x}}{\partial y}-\frac{1}{v^{2}} \frac{\partial B_{z}}{\partial t}\right)-i e_{4} \frac{1}{v}\left(-\frac{\partial \phi}{\partial x}-\frac{\partial B_{z}}{\partial y}+\frac{\partial B_{y}}{\partial z}-\frac{\partial A_{x}}{\partial t}\right) \\
& -i e_{5} \frac{1}{v}\left(-\frac{\partial \phi}{\partial y}-\frac{\partial B_{x}}{\partial z}+\frac{\partial B_{z}}{\partial x}-\frac{\partial A_{y}}{\partial t}\right)-i e_{6} \frac{1}{v}\left(-\frac{\partial \phi}{\partial z}-\frac{\partial B_{y}}{\partial x}+\frac{\partial B_{x}}{\partial y}-\frac{\partial A_{z}}{\partial t}\right)
\end{aligned}
$$

under the Lorentz Gauge condition

$$
\begin{aligned}
& \vec{\nabla} \cdot \vec{A}+\frac{1}{v^{2}} \frac{\partial \phi}{\partial t}=0 \\
& \vec{\nabla} \cdot \vec{B}+\frac{1}{v^{2}} \frac{\partial \varphi}{\partial t}=0
\end{aligned}
$$


then equation 23 reduced in the following octonion form

$\bar{\square} \mathbb{V}=\mathbb{F}$.

where $\mathbb{F}$ is generalized electromagnetic field of octonion of the dyon in isotropic medium

$$
\mathbb{F}=e_{0} F_{0}+e_{1} F_{1}+e_{2} F_{2}+e_{3} F_{3}+e_{4} F_{4}+e_{5} F_{5}+e_{6} F_{6}+e_{7} F_{7}
$$

where

$$
\begin{aligned}
& F_{0}=F_{7}=0 ; \\
& F_{1}=\left(-\frac{\partial \varphi}{\partial x}+\frac{\partial A_{z}}{\partial y}-\frac{\partial A_{y}}{\partial z}-\frac{1}{v^{2}} \frac{\partial B_{x}}{\partial t}\right) ; \\
& F_{2}=\left(-\frac{\partial \varphi}{\partial y}+\frac{\partial A_{x}}{\partial z}-\frac{\partial A_{z}}{\partial z x}-\frac{1}{v^{2}} \frac{\partial B_{y}}{\partial t}\right) ; \\
& F_{3}=\left(-\frac{\partial \varphi}{\partial z}+\frac{\partial A_{y}}{\partial x}-\frac{\partial A_{x}}{\partial y}-\frac{1}{v^{2}} \frac{\partial B_{z}}{\partial t}\right) ; \\
& F_{4}=-\frac{i}{v}\left(-\frac{\partial \phi}{\partial x}-\frac{\partial B_{z}}{\partial y}+\frac{\partial B_{y}}{\partial z}-\frac{\partial A_{x}}{\partial t}\right) ; \\
& F_{5}=-\frac{i}{v}\left(-\frac{\partial \phi}{\partial y}-\frac{\partial B_{x}}{\partial z}+\frac{\partial B_{z}}{\partial x}-\frac{\partial A_{y}}{\partial t}\right) ; \\
& F_{6}=-\frac{i}{v}\left(-\frac{\partial \phi}{\partial z}-\frac{\partial B_{y}}{\partial x}+\frac{\partial B_{x}}{\partial y}-\frac{\partial A_{z}}{\partial t}\right) ;
\end{aligned}
$$

in term of electric and magnetic field, we may described the different components of $\mathbb{F}$ as

$$
\begin{array}{ll}
F_{1} \longmapsto \mathcal{B}_{x} ; & F_{4} \longmapsto-i \frac{\mathcal{E}_{x}}{v} ; \\
F_{2} \longmapsto \mathcal{B}_{y} ; & F_{5} \longmapsto-i \frac{\mathcal{E}_{y}}{v} ; \\
F_{3} \longmapsto \mathcal{B}_{z} ; & F_{6} \longmapsto-i \frac{\mathcal{E}_{z}}{v} .
\end{array}
$$

Thus, from equation 28 the generalized electromagnetic field of dyons $\mathbb{F}$ in terms of the octonion in isotropic medium may be expressed as

$$
\begin{aligned}
\mathbb{F} & =e_{1}\left(\mathcal{B}_{x}+i e_{7} \frac{\mathcal{E}_{x}}{v}\right)+e_{2}\left(\mathcal{B}_{y}+i e_{7} \frac{\mathcal{E}_{y}}{v}\right)+e_{3}\left(\mathcal{B}_{z}+i e_{7} \frac{\mathcal{E}_{z}}{v}\right) \\
& =e_{1} \Psi_{x}+e_{2} \Psi_{y}+e_{3} \Psi_{z}
\end{aligned}
$$

where $\vec{\Psi}=\overrightarrow{\mathcal{B}}+i e_{7} \frac{\overrightarrow{\mathcal{\varepsilon}}}{v}$ is the generalized vector field of dyon in in isotropic medium [17, 18]. Now applying the differential 
operator to the equation 30 , we get

$$
\begin{aligned}
\square \mathbb{F}= & -e_{0}(\vec{\nabla} \cdot \overrightarrow{\mathcal{B}})+e_{1}\left[(\vec{\nabla} \times \overrightarrow{\mathcal{B}})_{x}-\frac{1}{v^{2}} \frac{\partial \mathcal{E}_{x}}{\partial t}\right] \\
& +e_{2}\left[(\vec{\nabla} \times \overrightarrow{\mathcal{B}})_{y}-\frac{1}{v^{2}} \frac{\partial \mathcal{E}_{y}}{\partial t}\right]+e_{3}\left[(\vec{\nabla} \times \overrightarrow{\mathcal{B}})_{z}-\frac{1}{v^{2}} \frac{\partial \mathcal{E}_{z}}{\partial t}\right] \\
& -i e_{4} \frac{1}{v}\left[(\vec{\nabla} \times \overrightarrow{\mathcal{E}})_{x}-\frac{\partial \mathcal{B}_{x}}{\partial t}\right]-i e_{5} \frac{1}{v}\left[(\vec{\nabla} \times \overrightarrow{\mathcal{E}})_{y}-\frac{\partial \mathcal{B}_{y}}{\partial t}\right] \\
& -i e_{6} \frac{1}{v}\left[(\vec{\nabla} \times \overrightarrow{\mathcal{E}})_{z}-\frac{\partial \mathcal{B}_{z}}{\partial t}\right]+i e_{7} \frac{1}{v}(\vec{\nabla} \cdot \overrightarrow{\mathcal{E}}) .
\end{aligned}
$$

the wave equation of dyons in isotropic medium in terms of octonion may be expressed as

$$
\bullet \mathbb{F}=\mathbb{J}
$$

where $\mathbb{J}$ is the generalized octonion current. In the isotropic medium generalized octonion current $\mathbb{J}$ is defined as

$$
\mathbb{J}=\mu\left(\mathrm{e}_{0} \varrho+\mathrm{e}_{1} \mathrm{j}_{\mathrm{x}}+\mathrm{e}_{2} \mathrm{j}_{\mathrm{y}}+\mathrm{e}_{3} \mathrm{j}_{\mathrm{z}}\right)-\frac{1}{\epsilon} \cdot \frac{\mathrm{i}}{\mathrm{v}}\left(\mathrm{e}_{4} \mathrm{k}_{\mathrm{x}}+\mathrm{e}_{5} \mathrm{k}_{\mathrm{y}}+\mathrm{e}_{6} \mathrm{k}_{\mathrm{z}}+\mathrm{e}_{7} \rho\right)
$$

Equations $(26)$ and $(32)$ are represents the octonion form of field equations associated respectively with the generalized potential and current of dyons in isotropic medium. Thus the equation (32) leads to the generalized Dirac Maxwell's equations of dyons in isotropic medium.

\section{Generalized Maxwell's field equations in Chiral Media}

In the case of homogeneous (isotropic) medium given by equation $\sqrt{1}$, we have taken the electric field $(\overrightarrow{\mathcal{E}})$ and the magnetic field $(\overrightarrow{\mathcal{B}})$ are time harmonic. The electric and magnetic fields in complex space can be defined as [15],

$$
\begin{aligned}
& \overrightarrow{\mathcal{E}}(x, t)=\operatorname{Re}\left\{\overrightarrow{\mathcal{E}}(x) e^{-i \omega t}\right\} \\
& \overrightarrow{\mathcal{B}}(x, t)=\operatorname{Re}\left\{\overrightarrow{\mathcal{B}}(x) e^{-i \omega t}\right\}
\end{aligned}
$$

Thus, the generalized Maxwell's equations (2) for dyons in homogeneous (isotropic) medium may be written as 


$$
\begin{aligned}
\vec{\nabla} \cdot \overrightarrow{\mathcal{E}} & =\frac{\rho(x)}{\epsilon} \\
\vec{\nabla} \cdot \vec{H} & =\varrho(x) \\
\nabla \times \overrightarrow{\mathcal{E}}(x) & =i \omega \overrightarrow{\mathcal{B}}(x)-\frac{\tilde{\mathrm{k}}(\mathrm{x})}{\epsilon} ; \\
\vec{\nabla} \times \vec{H}(x) & =-i \omega \vec{D}(x)+\mu \overrightarrow{\mathrm{j}}(x) .
\end{aligned}
$$

In chiral medium [19], the electric and magnetic fields are paired with each other. As such that the constitutive relations $\vec{D}=\epsilon \overrightarrow{\mathcal{E}}$ and $\overrightarrow{\mathcal{B}}=\mu \vec{H}$ will become in paired form as

$$
\begin{array}{lll}
\vec{D}=\epsilon \overrightarrow{\mathcal{E}}+\epsilon^{\prime} \vec{H} & \Longrightarrow & \vec{D}=\epsilon(\overrightarrow{\mathcal{E}}+\beta(\nabla \times \overrightarrow{\mathcal{E}})) \\
\overrightarrow{\mathcal{B}}=\mu \vec{H}+\mu^{\prime} \overrightarrow{\mathcal{E}} \quad \Longrightarrow & \overrightarrow{\mathcal{B}}=\mu(\vec{H}+\beta(\nabla \times \vec{H})) .
\end{array}
$$

where $\beta$ is chiral parameter and $\epsilon^{\prime}$ and $\mu^{\prime}$ are pairing constants and the relation (36) and (37) are known as Drude - Born - Fedorov constitutive relations [20, 21]. The cartesian field components of the equations (36), (37) are given by

$$
\begin{aligned}
& \overrightarrow{D_{x}}=\epsilon \mathcal{E}_{x}-\epsilon \beta(\nabla \times \mathcal{E})_{x} \\
& \overrightarrow{D_{y}}=\epsilon \mathcal{E}_{y}-\epsilon \beta(\nabla \times \mathcal{E})_{y} \\
& \overrightarrow{D_{z}}=\epsilon \mathcal{E}_{z}-\epsilon \beta(\nabla \times \mathcal{E})_{z} \\
& \overrightarrow{\mathcal{B}_{x}}=\mu H_{x}-\mu \beta(\nabla \times H)_{x} \\
& \overrightarrow{\mathcal{B}_{y}}=\mu H_{y}-\mu \beta(\nabla \times H)_{y} \\
& \overrightarrow{\mathcal{B}_{z}}=\mu H_{z}-\mu \beta(\nabla \times H)_{z} .
\end{aligned}
$$

using the Drude - Born - Fedorov constitutive relations (36) [37), the generalized Maxwell's equations (2) may be written as

$$
\begin{aligned}
\vec{\nabla} \cdot \overrightarrow{\mathcal{E}} & =\frac{\rho(x)}{\epsilon} \\
\vec{\nabla} \cdot \vec{H} & =\varrho(x) \\
\nabla \times \overrightarrow{\mathcal{E}}(x) & =+i \omega \mu(\vec{H}(x)+\beta(\nabla \times \vec{H}(x)))-\frac{\tilde{\mathrm{k}}(\mathrm{x})}{\epsilon} \\
\vec{\nabla} \times \vec{H}(x) & =-i \omega \epsilon(\overrightarrow{\mathcal{E}}(x)+\beta(\nabla \times \overrightarrow{\mathcal{E}}(x)))+\mu \overrightarrow{\mathrm{j}}(x)
\end{aligned}
$$

The equation (39) represents the Generalized Dirac-Maxwell's (GDM) field equations of dyons in chiral media. 


\section{Octonion Electrodynamic in Chiral Medium}

In the case of octonions the Born - Fedorov constitutive relations given by equation (36) (37) may be written as

$$
\overrightarrow{\mathcal{B}}=\left(\overrightarrow{\mathcal{B}_{x}}, \overrightarrow{\mathcal{B}_{y}}, \overrightarrow{\mathcal{B}_{z}}\right), \quad \vec{D}=\left(\overrightarrow{D_{x}}, \overrightarrow{D_{y}}, \overrightarrow{D_{z}}\right)
$$

where

$$
\begin{aligned}
& \overrightarrow{\mathcal{B}}_{x}=\mu H_{x} e_{1}-e_{7} \mu \beta(\nabla \times H)_{x} \cdot e_{4} ; \\
& \overrightarrow{\mathcal{B}}_{y}=\mu H_{y} e_{2}-e_{7} \mu \beta(\nabla \times H)_{y} \cdot e_{5} ; \\
& \overrightarrow{\mathcal{B}}_{z}=\mu H_{z} e_{3}-e_{7} \mu \beta(\nabla \times H)_{z} \cdot e_{6} ;
\end{aligned}
$$

equation (41) may be generalized as

$$
\overrightarrow{\mathcal{B}}=\mu \vec{H} e_{j}-e_{7} \mu \beta(\nabla \times H) \cdot e_{j+3} ; \quad(j=1,2,3)
$$

similarly

$$
\begin{aligned}
& \overrightarrow{D_{x}}=\epsilon \mathcal{E}_{x} e_{1}-e_{7} \epsilon \beta(\nabla \times \mathcal{E})_{x} \cdot e_{4} ; \\
& \overrightarrow{D_{y}}=\epsilon \mathcal{E}_{y} e_{2}-e_{7} \epsilon \beta(\nabla \times \mathcal{E})_{y} \cdot e_{5} ; \\
& \overrightarrow{D_{z}}=\epsilon \mathcal{E}_{z} e_{3}-e_{7} \epsilon \beta(\nabla \times \mathcal{E})_{z} \cdot e_{6} ;
\end{aligned}
$$

equation (43) written in the following reduced form

$$
\vec{D}=\epsilon \overrightarrow{\mathcal{E}} e_{j}-e_{7} \epsilon \beta(\nabla \times \mathcal{E}) \cdot e_{j+3} ; \quad(j=1,2,3) .
$$

generalized electromagnetic field of dyons $\overrightarrow{\mathcal{F}}$ of octonion in chiral medium then may be write as

$$
\overrightarrow{\mathcal{F}}=\overrightarrow{\mathcal{B}}+i e_{7} \vec{D}
$$

in this field equation we substitute $\overrightarrow{\mathcal{B}}$ and $\vec{D}$ value from equations 42 and 44 as 


$$
\overrightarrow{\mathcal{F}}=\left(\mu \vec{H} e_{j}-e_{7} \mu \beta(\nabla \times H) \cdot e_{j+3}\right)+i e_{7}\left(\epsilon \overrightarrow{\mathcal{E}} e_{j}-e_{7} \epsilon \beta(\nabla \times \mathcal{E}) \cdot e_{j+3}\right) ;(j=1,2,3)
$$

the equation 46 express as

$$
\begin{aligned}
\overrightarrow{\mathcal{F}} & =\left(\mu H_{x}+i e_{7} \epsilon \mathcal{E}_{x}\right) \cdot e_{1}+\left(\mu H_{y}+i e_{7} \epsilon \mathcal{E}_{y}\right) \cdot e_{2} \\
& +\left(\mu H_{z}+i e_{7} \epsilon \mathcal{E}_{z}\right) \cdot e_{3}-e_{7} \beta\left(\mu(\nabla \times H)_{x}+i e_{7} \epsilon(\nabla \times \mathcal{E})_{x}\right) \cdot e_{4} \\
& -e_{7} \beta\left(\mu(\nabla \times H)_{y}+i e_{7} \epsilon(\nabla \times \mathcal{E})_{y}\right) \cdot e_{5}-e_{7} \beta\left(\mu(\nabla \times H)_{z}+i e_{7} \epsilon(\nabla \times \mathcal{E})_{z}\right) \cdot e_{6}
\end{aligned}
$$

thus we may write equation 47 simple form as

$$
\begin{aligned}
\overrightarrow{\mathcal{F}} & =\left\{\mu H_{x}+\mu \beta(\nabla \times H)_{x}\right\} \cdot e_{1} \\
& +\left\{\mu H_{y}+\mu \beta(\nabla \times H)_{y}\right\} \cdot e_{2} \\
& +\left\{\mu H_{z}+\mu \beta(\nabla \times H)_{z}\right\} \cdot e_{3} \\
& +\left\{\epsilon \mathcal{E}_{x}+\epsilon \beta(\nabla \times \mathcal{E})_{x}\right\} \cdot e_{4} \\
& +\left\{\epsilon \mathcal{E}_{y}+\epsilon \beta(\nabla \times \mathcal{E})_{y}\right\} \cdot e_{5} \\
& +\left\{\epsilon \mathcal{E}_{z}+\epsilon \beta(\nabla \times \mathcal{E})_{z}\right\} \cdot e_{6}
\end{aligned}
$$

now operate $\square$ to the chiral octonion field $\overrightarrow{\mathcal{F}}$, we get

$$
\begin{aligned}
\square \mathcal{F} & =-\left[\mu \frac{\partial}{\partial x} H_{x}+\mu \beta \frac{\partial}{\partial x}(\nabla \times H)_{x}+\mu \frac{\partial}{\partial y} H_{y}+\mu \beta \frac{\partial}{\partial y}(\nabla \times H)_{y}+\mu \frac{\partial}{\partial z} H_{z}+\mu \beta \frac{\partial}{\partial z}(\nabla \times H)_{z}\right] \cdot e_{0} \\
& +\left[\mu \frac{\partial}{\partial y} H_{z}+\mu \beta \frac{\partial}{\partial y}(\nabla \times H)_{z}-\mu \frac{\partial}{\partial z} H_{y}-\mu \beta \frac{\partial}{\partial z}(\nabla \times H)_{y}-\epsilon \frac{\partial}{\partial t} \mathcal{E}_{x}-\epsilon \beta \frac{\partial}{\partial t}(\nabla \times \mathcal{E})_{x}\right] \cdot e_{1} \\
& +\left[\mu \frac{\partial}{\partial z} H_{x}+\mu \beta \frac{\partial}{\partial z}(\nabla \times H)_{x}-\mu \frac{\partial}{\partial x} H_{z}-\mu \beta \frac{\partial}{\partial x}(\nabla \times H)_{z}-\epsilon \frac{\partial}{\partial t} \mathcal{E}_{y}-\epsilon \beta \frac{\partial}{\partial t}(\nabla \times \mathcal{E})_{y}\right] \cdot e_{2} \\
& +\left[\mu \frac{\partial}{\partial x} H_{y}+\mu \beta \frac{\partial}{\partial x}(\nabla \times H)_{y}-\mu \frac{\partial}{\partial y} H_{x}-\mu \beta \frac{\partial}{\partial y}(\nabla \times H)_{x}-\epsilon \frac{\partial}{\partial t} \mathcal{E}_{z}-\epsilon \beta \frac{\partial}{\partial t}(\nabla \times \mathcal{E})_{z}\right] \cdot e_{3} \\
& +i\left[\epsilon \frac{\partial}{\partial z} \mathcal{E}_{y}+\epsilon \beta \frac{\partial}{\partial z}(\nabla \times \mathcal{E})_{y}-\epsilon \frac{\partial}{\partial y} \mathcal{E}_{z}-\epsilon \beta \frac{\partial}{\partial y}(\nabla \times \mathcal{E})_{z}-\mu \frac{\partial}{\partial t} H_{x}-\mu \beta \frac{\partial}{\partial t}(\nabla \times H)_{x}\right] \cdot e_{4} \\
& +i\left[\epsilon \frac{\partial}{\partial x} \mathcal{E}_{z}+\epsilon \beta \frac{\partial}{\partial x}(\nabla \times \mathcal{E})_{z}-\epsilon \frac{\partial}{\partial z} \mathcal{E}_{x}-\epsilon \beta \frac{\partial}{\partial z}(\nabla \times \mathcal{E})_{x}-\mu \frac{\partial}{\partial t} H_{y}-\mu \beta \frac{\partial}{\partial t}(\nabla \times H)_{y}\right] \cdot e_{5} \\
& +i\left[\epsilon \frac{\partial}{\partial y} \mathcal{E}_{x}+\epsilon \beta \frac{\partial}{\partial y}(\nabla \times \mathcal{E})_{x}-\epsilon \frac{\partial}{\partial x} \mathcal{E}_{y}-\epsilon \beta \frac{\partial}{\partial x}(\nabla \times \mathcal{E})_{y}-\mu \frac{\partial}{\partial t} H_{z}-\mu \beta \frac{\partial}{\partial t}(\nabla \times H)_{z}\right] \cdot e_{6} \\
& +i\left[\epsilon \frac{\partial}{\partial x} \mathcal{E}_{x}+\epsilon \beta(\nabla \times \mathcal{E})_{x}+\epsilon \frac{\partial}{\partial y} \mathcal{E}_{y}+\epsilon \beta(\nabla \times \mathcal{E})_{y}+\epsilon \frac{\partial}{\partial z} \mathcal{E}_{z}+\epsilon \beta(\nabla \times \mathcal{E})_{z}\right] \cdot e_{7}
\end{aligned}
$$

equation 49 may write in the following reduced form as 


$$
\begin{aligned}
\ominus \mathcal{F}= & -[\mu(\vec{\nabla} \cdot \vec{H})+\mu \beta \vec{\nabla} \cdot(\vec{\nabla} \times \vec{H})] \cdot e_{1} \\
& +\left[\mu(\vec{\nabla} \times \vec{H})-\epsilon \frac{\partial}{\partial t} \overrightarrow{\mathcal{E}}+\mu \beta \vec{\nabla} \cdot(\vec{\nabla} \times \vec{H})-\epsilon \beta \frac{\partial}{\partial t}(\vec{\nabla} \times \overrightarrow{\mathcal{E}})-\mu \beta \vec{\nabla} \cdot(\vec{\nabla} \times \vec{H})\right] \cdot e_{j} \\
& +i\left[-\epsilon(\vec{\nabla} \times \overrightarrow{\mathcal{E}})+\epsilon \beta \vec{\nabla} \cdot(\vec{\nabla} \times \overrightarrow{\mathcal{E}})-\mu \frac{\partial}{\partial t} \vec{H}-\mu \beta \frac{\partial}{\partial t}(\vec{\nabla} \times \vec{H})-\epsilon \beta \vec{\nabla} \cdot(\vec{\nabla} \times \overrightarrow{\mathcal{E}})\right] \cdot e_{j+3} \\
& +i[\epsilon(\vec{\nabla} \cdot \overrightarrow{\mathcal{E}})+\epsilon \beta \vec{\nabla} \cdot(\vec{\nabla} \times \overrightarrow{\mathcal{E}})] \cdot e_{7}
\end{aligned}
$$

The octonion GDM wave equation in Chiral medium of dyons may be written as

$$
\square \mathcal{F}=\mathcal{J}
$$

where octonion current source $\mathcal{J}$ is

$$
\begin{aligned}
\mathcal{J} & =e_{0} J_{0}+e_{1} J_{1}+e_{2} J_{2}+e_{3} J_{3}+e_{4} J_{4}+e_{5} J_{5}+e_{6} J_{6}+e_{7} J_{7} \\
& =-e_{0} \varrho+e_{j} \vec{j}-i e_{j+3} \vec{k}+i e_{7} \rho
\end{aligned}
$$

from the equation (51) we get following relation

$$
\begin{aligned}
\epsilon(\vec{\nabla} \cdot \overrightarrow{\mathcal{E}}) & =\rho ; \\
\mu(\vec{\nabla} \cdot \vec{H}) & =\varrho ; \\
\epsilon(\vec{\nabla} \times \overrightarrow{\mathcal{E}}) & =-\mu \frac{\partial}{\partial t} \vec{H}-\mu \beta \frac{\partial}{\partial t}(\vec{\nabla} \times \vec{H})-\vec{k} \\
\mu(\vec{\nabla} \times \vec{H}) & =\epsilon \frac{\partial}{\partial t} \overrightarrow{\mathcal{E}}+\epsilon \beta \frac{\partial}{\partial t}(\vec{\nabla} \times \overrightarrow{\mathcal{E}})+\vec{j} .
\end{aligned}
$$

These equations are octonionic GDM equations in Chiral medium. If we use the time-derivative relations as

$$
\begin{aligned}
& \frac{\partial}{\partial t} \vec{D}=\epsilon \frac{\partial}{\partial t}(\overrightarrow{\mathcal{E}}+\beta(\vec{\nabla} \times \overrightarrow{\mathcal{E}}))=\epsilon \frac{\partial}{\partial t} \overrightarrow{\mathcal{E}}+\epsilon \beta \frac{\partial}{\partial t}(\vec{\nabla} \times \overrightarrow{\mathcal{E}}) \\
& \frac{\partial}{\partial t} \overrightarrow{\mathcal{B}}=\mu \frac{\partial}{\partial t}(\vec{H}+\beta(\vec{\nabla} \times \vec{H}))=\mu \frac{\partial}{\partial t} \vec{H}+\mu \beta \frac{\partial}{\partial t}(\vec{\nabla} \times \vec{H})
\end{aligned}
$$

and we have

$$
\begin{aligned}
& \vec{\nabla} \cdot \vec{D}=\epsilon \vec{\nabla} \cdot(\overrightarrow{\mathcal{E}}+\beta(\vec{\nabla} \times \overrightarrow{\mathcal{E}}))=\epsilon(\vec{\nabla} \cdot \overrightarrow{\mathcal{E}}) \\
& \vec{\nabla} \cdot \overrightarrow{\mathcal{B}}=\mu \vec{\nabla} \cdot(\vec{H}+\beta(\vec{\nabla} \times \vec{H}))=\mu(\vec{\nabla} \cdot \vec{H})
\end{aligned}
$$


using above equations (54), (55) and equations $(56),(57)$, we get

$$
\begin{aligned}
\epsilon(\vec{\nabla} \cdot \overrightarrow{\mathcal{E}}) & =\rho ; \\
\mu(\vec{\nabla} \cdot \vec{H}) & =\varrho ; \\
\epsilon(\vec{\nabla} \times \overrightarrow{\mathcal{E}}) & =-\frac{\partial}{\partial t} \overrightarrow{\mathcal{B}}-\vec{k} ; \\
\mu(\vec{\nabla} \times \vec{H}) & =\frac{\partial}{\partial t} \vec{D}+\vec{j} .
\end{aligned}
$$

There equations are another form of Maxwell's equation in Chiral medium in case of generalized octonion electrodynamics.

\section{Discussion}

The equations $([42)$ and $(44)$ showing the constitutive relation and then describe the rich variety of physical phenomenon representing the properties and responses of the chiral medium and to the application of generalized octonionic electromagnetic field of dyons. The octonions field equations are described here in isotropic medium and chiral medium. The present theory of generalized octonions electrodynamics of dyons leads to the connection between the mechanical parameters with the chirality and dielectric properties. And we formulised octonions Maxwell's equation for electromagnetic wave theory, are being unique, compact, and consistent manner for isotropic and chiral medium.

\section{References}

[1] L. E. Dickson, "On Quaternions and their Generalization and the History of the Eight Square Theorem", Ann. Math. 20, (1919) 155 .

[2] J. C. Baez, "The Octonions", Bull. Am. Math. Soc. 39, (2001) 145.

[3] G. t' Hooft, “Magnetic Monopoles in Unified Gauge Theories”, Nucl. Phys. B79, (1974) 276.

[4] A. M. Polyakov, "Particle Spectrum in Quantum Field Theory”, JEPT Letter, 20, (1974) 194.

[5] B. Julia and A. Zee, "Poles with both Magnetic and Electric Charges in non-Abelian Gauge Theory", Phys. Rev. D11, (1975) 2227.

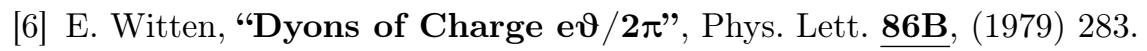

[7] P. S. Bisht, O. P. S. Negi and B. S. Rajput, "Quaternion Gauge Theory of Dyonic Fields", Prog. Theor. Phys. 85, (1991) 157.

[8] P. S. Bisht, O. P. S. Negi and B. S. Rajput, "Null tetrad Formulation of non-Abelian Dyons", Int. J. Theor. Phys. 32, (1993) 2099.

[9] V. V. Kravchenko, "Applied Quaternionic Analysis, Research and Exposition in Mathematics", Heldermann Verlage, Germany , 28 (2003). 
[10] V. V. Kravchenko, “Quaternionic Reformulation of Maxwell's Equations for Inhomogeneous Media and New Solutions", Zeitschrift Fur Analysis and Anwenduhgen, 21, (2002) 21.

[11] K. V. Khmelnytskaya and V. V. Kravchenko, "Quaternionic Integral Representations for Electromagnetic Fields in Chiral Media", Zeitschrift Fur Analysis and Anwenduhgen, 56, (2001) 4641.

[12] S. M. Grudsky, K. V. Khmelnytskaya and V. V. Kravchenko, “On a Quaternionic Maxwell Equation for the Time-dependent Electromagnetic Field in a Chiral Medium" J. Phys. A. Math. Gen., 37, (2004) 4641.

[13] Jivan Singh, P. S. Bisht and O. P. S. Negi, "Quaternion Analysis of Generalized Fields of Dyons in Isotropic Medium", J. Phys. A: Math. and Gen., (2007).

[14] Jivan Singh, P. S. Bisht and O. P. S. Negi, "Quaternion Analyticity of Time-Harmonic Dyon Field Equations", arXiv: hep-th/0703107 (2007).

[15] Jivan Singh, P. S. Bisht and O. P. S. Negi, "Generalized Electromagnetic Fields in Chiral Medium", J. Phys. A: Math. and Gen., (2007).

[16] P. A. M. Dirac, "Quantized Singularities in the Electromagnetic Field", Proc. R. Soc. London Sec , A60, (1931) 133 .

[17] B. C. Chanyal, P. S. Bisht, O. P. S. Negi, "Generalized Octonion Electrodynamics", Int. J. Theor. Phys. 49, (2010) 1333.

[18] B. C. Chanyal, P. S. Bisht, O. P. S. Negi, "Generalized Split - Octonion Electrodynamics", Int. J. Theor. Phys. 50, (2011) 1919; “Octonion Quantum Chromodynamics”, Int. J. Theor. Phys. 51 (2012) 3410.

[19] M. Tanisli and M. E. Kansu, “ Octonionic Maxwell's Equations for Bi-Isotropic Media”, J. Math. Phys. 52, (2011) 053511.

[20] A. Lakhtakia and Beltrami, "Fields in Chiral Media", World Scientific, Singapore (1994).

[21] I. V. Lindell, A. H. Sihvola, S. A. Tretyakov and A. J. Viitanen, "Electromagnetic Wave in Chiral and BiIsotropic Media", Artech House Publisher, Boston, MA (1994). 